\title{
Cooperação, comportamentos proativos ou simplesmente cidadania organizacional? Uma revisão da produção nacional na área
} Cooperation, proactive behaviors, or simply organizational citizenship? A review of Brazilian production in the area Cooperación, conductas proactivas o simplemente ciudadanía organizacional? Una revisión de la producción nacional en el área

\author{
Clara CANTAL ${ }^{1, \text { a }}$ \\ Jairo E. BORGES-ANDRADE \\ Juliana B. PORTO ${ }^{b}$ \\ Victoria University of Wellington, Wellington, Nova Zelândia ${ }^{a}$, \\ Universidade de Brasília, Brasília, DF, Brasil
}

Resumo

O conceito de cidadania organizacional guarda uma história teórica de 37 anos e uma empírica de 31, e sua importância é destacada por pesquisadores e profissionais internacionalmente. Nos anos 2000, aumentaram os números de metanálises internacionais abordando o tema, de artigos revisados por essas metanálises e de estudos de cidadania organizacional como um construto multidimensional. Tendo em vista o histórico e o volume de produção, este artigo buscou revisar a produção de artigos empíricos nacionais em cidadania organizacional de 1996 a 2013. Foram encontrados apenas oito artigos em cinco dos 14 periódicos avaliados das áreas de administração e psicologia. Dos artigos encontrados, $75 \%$ ocupou-se do estudo de cidadania organizacional como um construto único composto por cinco dimensões, e foram utilizadas duas diferentes escalas para mensurá-lo. Os resultados da revisão sugerem que o número de publicações brasileiras na área precisa aumentar, assim como o uso de diferentes técnicas de coleta de dados e pesquisas que avaliem a relação entre cidadania organizacional e seus consequentes.

Palavras-chave:

Cidadania organizacional; revisão nacional; artigos empíricos.

Abstract The term Organizational Citizenship holds a theoretical history of 37 years and an empirical history of 31 years. Its importance is highlighted by researchers and professionals internationally. In the decade of 2000, the number of international meta-analyses addressing the issue increased, as well as the number of articles reviewed in these meta-analyses and the study of Organizational Citizenship as a multidimensional construct. Given the history and the volume of international production on the theme, this article sought to review the Brazilian production of empirical articles about Organizational Citizenship from 1996 to 2013. Only eight articles were found in five of the 14 journals on Administration and Psychology evaluated. Three quarters of the articles studied Organizational Citizenship as a single construct composed of five dimensions. In these articles, two different scales were used to measure it. The review results suggest that the number of Brazilian publications in the area needs to increase, as well as the use of different data collection and research techniques to assess the relationship between Organizational Citizenship and its consequents.

Keywords:

Organizational citizenship; national review; empirical articles. 
La expresión Ciudadanía Organizacional tiene una historia teórica de 37 años y una historia empírica de 31 años y su importancia es destacada por investigadores y profesionales a nivel internacional. En los años 2000, se incrementó el número de meta-análisis que abordan el tema, así como el número de artículos revisados en este tipo de trabajo y el estudio de ciudadanía organizacional como un constructo multidimensional. Teniendo en cuenta la historia y el volumen de la producción internacional sobre el tema, este artículo buscó revisar la producción nacional de artículos empíricos sobre Ciudadanía Organizacional desde 1996 hasta 2013. Sólo ocho artículos fueron encontrados en cinco de las 14 revistas de Administración y Psicología evaluadas. De los artículos encontrados, 75\% estudiaron el tema Ciudadanía Organizacional como un único constructo compuesto de cinco dimensiones. En estos artículos, se utilizaron dos escalas diferentes para medirlo. Los resultados de la revisión sugieren que el número de publicaciones brasileñas en el área debe aumentar, así como el uso de diferentes técnicas de colecta de datos y de investigación que evaluen la relación entre la ciudadanía organizacional y sus consecuentes.

Palabras-clave:

Ciudadanía organizacional; revisión nacional; artículos empíricos.

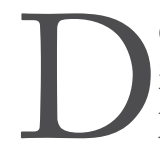

esde seu surgimento na década de 1960, a área de comportamento organizacional tem enfrentado inúmeras dificuldades em relação à sua conceituação, à determinação de seus níveis estruturais, à delimitação dos temas tratados sob sua alçada e às disciplinas que norteiam sua compreensão (Siqueira, 2002). De forma similar, resultando ou não dessas dificuldades, a realidade de muitas de suas subáreas, como a de cidadania organizacional, tem sido a mesma.

Apesar da introdução do conceito de cidadania organizacional na literatura há 37 anos, da publicação do primeiro artigo empírico envolvendo essa variável há 31 anos e da reconhecida importância por pesquisadores da área, falta ao conceito a delimitação de seus constituintes (Moon, Van Dyne, \& Wrobel, 2004; Rego, 2002). $\mathrm{O}$ foco da literatura internacional tem recaído antes em suas relações com outros construtos organizacionais do que em seus constituintes, o que tem prejudicado bastante o estudo de um construto comum com propriedades semelhantes em diferentes pesquisas. Mesmo assim, essa literatura já indica que ele tem importância prática ao apresentar evidências empíricas sobre suas associações com os desempenhos individual e organizacional (Dalal, 2005; Nielsen, Hrivnak, \& Shaw, 2009; Podsakoff, Witing, Podsakoff, \& Blume, 2009).

Tendo em vista a relação entre cidadania organizacional e variáveis de desempenho individual e organizacional, além da ausência de uma revisão nacional sobre o tema e da inclusão dos estudos nacionais nas metanálises encontradas, buscou-se revisar a produção brasileira em cidadania organizacional entre os anos de 1996 e 2013, no nível micro de análise. É relevante compará-la à produção internacional, uma vez que o tema em foco pode ser suscetível a influências da cultura nacional (Gelfand, Erez, \& Aycan, 2007).

A partir dessa revisão, espera-se traçar uma agenda de estudo para o desenvolvimento do tema no Brasil. Primeiramente será feita uma breve descrição do construto cidadania organizacional e de sua investigação descrita em revisões internacionais. Serão, então, apresentados o método, os resultados e a discussão para a revisão bibliográfica de artigos nacionais na área. Por fim, serão introduzidas as limitações dessa produção e proposta uma agenda de estudo tendo em vista suas características.

Muito embora comportamentos de cidadania organizacional sejam tratados na literatura científica há mais de 30 anos, ainda não existe unanimidade quanto às dimensões ou aos comportamentos específicos que o constituem, o que gera confusão em sua diferenciação em relação a outros construtos. Organ (1988, citado por Podsakoff, MacKenzie, Paine, \& Bachrach, 2000), um dos propulsores da pesquisa na área, definiu comportamentos de cidadania organizacional como:

Comportamentos individuais discricionários, não reconhecidos explicitamente ou diretamente pelo sistema de recompensa formal, e que, quando agregados, promovem o efetivo funcionamento da organização. Como papel ou descrição de papel, concebemos que esses comportamentos não são exigências forçadas ao papel ou à descrição de trabalho, o que significa que não fazem parte do que está estipulado de forma clara e específica em termos do contrato de trabalho da pessoa com a organização; o comportamento é antes uma questão de escolha própria, já que sua omissão geralmente não é compreendida como punível. (Podsakoff et al., 2000, p. 513) 
A definição descrita pelo autor aponta diferenças tangíveis entre esse construto e o de responsabilidade social, com o qual é muitas vezes confundido. Enquanto cidadania organizacional ocupa-se de comportamentos individuais e discricionários, o de responsabilidade social ocupa-se de comportamentos organizacionais planejados. O foco de responsabilidade social está na preocupação da organização com o tratamento ético e responsável prestado a seus stakeholders, tanto dentro quanto fora da organização, e na criação de padrões mais elevados de vida para eles (Hopkins, 2003). Esses stakeholders podem variar de funcionários da própria organização, beneficiados por políticas éticas de recursos humanos, a desabrigados na comunidade em que a organização está inserida, que se beneficiam de um treinamento ofertado por ela, como adiciona Hopkins (2005).

Diferente de responsabilidade social, variável macro-organizacional ligada ao reconhecimento e à apreciação de funcionários e não funcionários pela organização, cidadania organizacional é uma variável microorganizacional que se refere a comportamentos dos funcionários direcionados aos colegas de trabalho ou à organização. A confusão na literatura parece advir da origem e do alvo dos comportamentos definidos em seus conceitos.

Autores na área também se esforçam em diferenciar cidadania organizacional de desempenho na tarefa. Cidadania organizacional não varia entre postos de trabalho em uma mesma organização, constituindo-se em comportamentos voluntários e cooperativos dos funcionários para com os colegas de trabalho e a organização. Desempenho na tarefa varia, sendo integralmente ligado aos comportamentos e às tarefas descritos para o posto de trabalho (Borman, Penner, Allen, \& Motowidlo, 2001). A confusão parece ser originada do caráter voluntário ou não do comportamento e de sua estabilidade ao longo da organização.

Embora a definição de cidadania organizacional de Organ (1988, citado por Podsakoff et al., 2000) tenha sido revisitada algumas vezes e dimensões tenham sido propostas para o construto, inclusive pelo próprio autor, a produção na área parece não ser unânime quanto aos seus componentes. A dificuldade na delimitação do construto e em sua diferenciação quanto a outros parece ser fruto da ampla gama de elementos sob seu guarda-chuva, o que impacta em sua definição, caracterizando cidadania organizacional mais pelo que ela não é, isto é, desempenho na tarefa e responsabilidade social.

Foram encontradas quase 30 formas diferentes de comportamentos de cidadania organizacional, agrupados em: (a) comportamento de ajuda, (b) espírito esportivo, (c) lealdade organizacional, (d) complacência organizacional, (e) iniciativa individual, (f) virtude cívica e (g) autodesenvolvimento (Podsakoff et al., 2000). Mesmo com essa ampla gama de formas, os estudos e teóricos da área assumem a necessidade do estudo da variável como um construto único, composto por diferentes dimensões relacionadas (Moon et al., 2004).

Utilizando o caminho inverso, ou seja, partindo de dimensões predefinidas de cidadania organizacional e posterior avaliação da diferenciação dessas dimensões em estudos da área, não foi possível diferenciar as dimensões propostas por Organ (1988, citado por Podsakoff et al., 2000), e mais utilizadas na literatura, altruísmo, espírito esportivo, conscienciosidade, cortesia e virtude cívica - devido às altas correlações entre elas e a não diferenciação quanto aos preditores (LePine, Erez, \& Johnson, 2002).

De acordo com Podsakoff et al. (2000), ocorreu também o crescimento de publicações internacionais na área, uma vez que foram publicados 13 artigos entre 1983 e 1988, enquanto foram publicados 122 artigos entre 1993 e 1998. Adicionalmente, a pesquisa com variáveis antecedentes do construto tem focalizado predominantemente características individuais ou do empregado (satisfação, justiça, comprometimento e confiança no líder), características da tarefa (feedback, rotinização e satisfação na tarefa), características organizacionais (formalização, inflexibilidade e apoio organizacionais, apoio consultivo aos funcionários, coesão do grupo, entre outros) e comportamentos do líder (liderança transformacional, articulação da visão de liderança, provisão de modelo apropriado, entre outros). Avaliações subjetivas e objetivas de desempenhos individual e organizacional foram variáveis consequentes relacionadas a ela, muito embora apenas a uma gama específica delas.

Diante do estudo de diversos comportamentos de cidadania organizacional, Moon et al. (2004) propuseram, com base na literatura, um modelo circumplexo para seus constituintes, que os alinha espacialmente em um círculo. $\mathrm{O}$ modelo proposto abarcou as dimensões de ajuda, espírito esportivo, complacência e inovação, e estabeleceu sua variação em torno de dois eixos: (a) promoção versus proteção e (b) interpessoal versus organizacional.

Nessa revisão de estudos empíricos, tendo por base o modelo referido, foram encontrados 164 artigos empíricos publicados entre 1983 e 2003. A revisão indicou ainda o aumento do número de artigos publicados e 
do estudo de cidadania organizacional como um construto único, e a diminuição do número de dimensões estudadas por artigo com o tempo. A frequência do estudo dos quadrantes de ajuda (combinação entre promoção e interpessoal), complacência (combinação entre proteção e organizacional) e espírito desportivo (combinação entre proteção e interpessoal) na literatura foi significativamente maior que o estudo de inovação (combinação entre promoção e organizacional) nos vários anos.

Por fim, constatou-se a utilização de diferentes escalas nos estudos que evidenciaram variadas dimensões de cidadania organizacional, como aquelas de Van Dyne, Vandewalle, Kostova, Latham e Cummings (2000), focando a dimensão de ajuda; de Pond, Nacoste, Mohr e Rodriguez (1997), focando as dimensões de ajuda e complacência; e de variados autores como Zellars, Tepper e Duffy (2002), focando as cinco dimensões propostas por Organ (1988, citado por Podsakoff et al., 2000).

As metanálises internacionais descritas anteriormente destacam o grande número de estudos que consideraram diferentes dimensões de cidadania organizacional. Contudo, ainda não parece evidente na literatura internacional as melhores maneiras teórica e empírica de dimensionar os comportamentos de cidadania organizacional, nem diferenças claras entre os modelos quanto ao relacionamento com outras variáveis do contexto organizacional. Neste sentido, há necessidade de maior unicidade no estudo do tema, independente de sua estruturação em um ou mais fatores, como apontam Moon et al. (2004).

Adicionalmente às metanálises sobre cidadania organizacional, uma parte delas, no âmbito internacional, que floresceu nos anos 2000, a relacionou com outras variáveis, como trabalho contraprodutivo, tensão emocional, relacionamento com o líder, resultados individuais e organizacionais, personalidade, estressores e desempenho no nível grupal (Chang, Johnson, \& Yang, 2007; Chiaburu, Oh, Berry, Li, \& Gardner, 2011; Dalal, 2005; Eatough, Chang, Miloslavic, \& Johnson, 2011; Ilies, Nahrgang, \& Morgeson, 2007; Nielsen et al., 2009; Podsakoff et al., 2009). Além disso, cidadania organizacional foi tratada em revisão do Annual Review of Psychology sobre comportamento organizacional transcultural (Gelfand et al., 2007), o que sugere sua variação de cultura para cultura e valida a importância de estudá-la nacionalmente.

Boa parte das revisões apontadas já mostra o interesse da área por variáveis de desempenho impactadas por cidadania organizacional e que, por definição, podem refletir em perdas ou ganhos monetários para a organização. Na revisão de Podsakoff et al. (2009), por exemplo, foram observadas correlações corrigidas de 0,47 entre desempenho na tarefa e cidadania organizacional voltada para outros indivíduos e 0,54 entre desempenho na tarefa e cidadania organizacional voltada para a organização. Na mesma metanálise, ao serem consideradas medidas do nível organizacional, cidadania organizacional teve uma correlação corrigida negativa de 0,52 com custos na unidade de trabalho.

A revisão da literatura internacional na área mostra desafios e aumento considerável da produção e da importância dada ao tema. Será que a produção nacional possui as mesmas características e enfrenta os mesmos desafios que a produção internacional? $\mathrm{O}$ construto, suas medidas e seus antecedentes e consequentes podem ser considerados equivalentes ao encontrado fora do Brasil para que os achados descobertos em outras culturas nacionais possam ser aplicados aqui?

$\mathrm{Na}$ literatura nacional, é possível verificar à primeira vista a quase inexistência de artigos que abordem cidadania organizacional como um todo - ao se utilizar a ferramenta de busca no site do portal Scielo, em fevereiro de 2014, buscando a expressão cidadania organizacional, só apareceram cinco referências. Entre elas, duas ${ }^{1}$ já constavam na revisão nacional aqui apresentada, e três continham amostras de outros países. Por isso, ressalta-se a importância de uma análise minuciosa dos artigos publicados, atentando-se aos constituintes do fenômeno, como feito nas revisões internacionais relatadas.

\section{MÉTODO}

A fim de analisar a produção nacional na área de cidadania organizacional, foram revisados relatos de pesquisas publicados em 14 periódicos de psicologia e administração (Tabela 1), segundo critérios e procedimentos descritos por Borges-Andrade e Pagotto (2010).

Almeida, S.P., \& Ferreira, M. C. (2010). O impacto das atitudes frente às mudanças organizacionais nos comportamentos de cidadania. Psicologia: Ciência e Profissão, 30(3), 492-504. doi: http://dx.doi.org/10.1590/S1414-98932010000300005

Siqueira, M. M. M. (2003). Proposição e análise de um modelo para comportamentos de cidadania organizacional. Revista de Administração Contemporânea, 7(Edição especial), 165-184. doi: http://dx.doi.org/10.1590/S1415-65552003000500009 
Periódicos de Psicologia

Estudos em Psicologia (UFRN)

Estudos em Psicologia (PUC Campinas)

Psico PUCRS

Psico USF

Psicologia, Ciência e Profissão (CFP)

Psicologia em Estudo (UEM)

Psicologia: Organizações e Trabalho - rPOT (SBPOT)

Psicologia: Reflexão e Crítica (UFRS)
Periódicos de Administração

Organizações e Sociedade - O\&S (UFBA)

Revista Administração USP - RAUSP

Revista Administração Contemporânea - RAC (ANPAD)

Revista Administração Empresas - RAE (FGV-SP)

Revista Administração Pública - RAP (FGV-RJ)

Psicologia: Teoria e Pesquisa (UnB)

Os artigos considerados foram aqueles publicados entre 1996 e 2013 que apresentassem relatos de resultados de pesquisas realizadas no Brasil e em que o objeto de estudo fosse cidadania organizacional, ou um de seus componentes no nível micro de análise (Staw, 1984). Além de cidadania organizacional como um todo, consideramos civismo, espontaneidade e comportamentos proativos e pró-sociais, como cooperação e ajuda.

Ressalta-se que os componentes avaliados nesta revisão não são os definidos por Moon et al. (2004), uma vez que a revisão começou anos antes e esses elementos ainda não estavam em voga na literatura nacional. Após a análise minuciosa dos artigos de acordo com os critérios mencionados, eles foram registrados e categorizados conforme as categorias adaptadas de Borges-Andrade e Pagotto (2010) presentes na Tabela 2.

TABELA 2. Critérios de análise adaptados de Borges-Andrade e Pagotto (2010)

Critérios de análise

Instituição de vínculo do(s) autor(es)

Produção ocorrida em programa de pós-

-graduação

stricto sensu

Grau de escolaridade do(s) autor(es)

Área de titulação do(s) autor(es)

Natureza do estudo

Desenho da investigação

Natureza da pesquisa

Finalidade do estudo

\section{Características analisadas}

Nome da organização de vinculação

Dissertação de Mestrado, Tese de Doutorado

Graduando, Graduado, Mestrando, Mestre, Doutorando, Doutor

Psicologia, Administração, outras

Pesquisa com desenho, relato de experiência

Survey, experimental e pesquisa-ação

Quantitativa, qualitativa, quali-quanti

Gerar conhecimento e/ou instrumento, e/ou tecnologia 


\author{
Natureza da amostragem \\ Origem dos dados coletados \\ Procedimento de coleta de dados \\ Procedimento de análise de dados \\ Setor estudado \\ Segmento da economia
}

Estudo de caso, de amostra (uma organização, várias organizações)

Primária e/ou secundária

Questionário/escala, entrevista, observação, análise documental, testes/prova, outros.

Estatística inferencial, descritiva, análise de conteúdo

Privado, público, terceiro setor, misto

Primário, secundário, terciário, diverso

Cabe ressaltar que o levantamento dos artigos por esses autores nesses periódicos é mais amplo e ocupa-se de comportamento organizacional como um todo e de todas as suas categorias, com base em revisões equivalentes publicadas no Annual Revierw of Psychology. Esse levantamento é feito e atualizado há anos, seguindo procedimentos e indicadores de classificação padronizados. Ademais, é fruto de uma parceria entre docentes e discentes de pós-graduação em psicologia na Universidade de Brasília, na Universidade Salgado de Oliveira (Campus de Niterói, RJ) e na Universidade Federal da Bahia.

\section{RESULTADOS}

No total foram encontrados oito artigos publicados entre 1996 e 2013 em cinco dos 14 periódicos avaliados. Considerando os elementos de cidadania organizacional avaliados nos artigos, seis ocuparam-se do estudo de um construto único composto por cinco dimensões, e dois ocuparam-se do estudo de cooperação ou comportamentos proativos (Tabela 3). As escalas de Porto e Tamayo (2003) e Siqueira (1995) foram utilizadas para mensurar as cinco dimensões do construto, e ambas baseiam a descrição de suas dimensões na definição apresentada por Katz e Kahn (1974).

TABELA 3. Elementos de cidadania organizacional considerados nos artigos avaliados

Elementos estudados $\quad N$

Cidadania organizacional como construto único com dimensões

Cooperação

Comportamentos proativos

Total
$N$

6

1

1

8

Os dados sugerem que a literatura nacional caminha para a unicidade desse construto, como proposto por Moon et al. (2004). As escalas utilizadas reconhecem nele as dimensões de sugestóes criativas ao sistema, proteção ao sistema, criação de clima favorável à organização no ambiente externo, autotreinamento e cooperação com os colegas. As dimensões corroboradas na literatura nacional por análise fatorial diferem daquelas observadas internacionalmente e empiricamente por Podsakoff et al. (2000) e Moon et al. (2004). Mesmo assim, os itens das escalas brasileiras guardam certa semelhança temática com as dimensões estabelecidas internacionalmente (Tabela 4). 
TABELA 4. Comparação entre as dimensões nacionais e internacionais de cidadania organizacional

\section{Escalas Brasileiras - Porto e Tamayo (2003) e Siqueira (1995)}

Dimensões

Sugestões criativas ao sistema

Proteção ao sistema

Criação de clima favorável à organização no ambiente externo

Autotreinamento

Cooperação com os colegas

\section{Descrição}

Agrega comportamentos de sugestão de novas ideias à administração para beneficiar a organização.

Considera ações que visam a zelar pelo patrimônio da organização.

Inclui comportamentos de divulgação das vantagens e dos méritos da organização fora do ambiente detrabalho.

Congrega comportamentos de busca de cursos e eventos que melhorem a sua performance no trabalho.

Inclui comportamentos de auxílio aos colegas de trabalho visando o benefício da organização.

Podsakoff et al. (2000)

Dimensões

Descrição

Comportamento de ajuda

Considera comportamentos de ajuda voluntária a outros na resolução de problemas relacionados ao trabalho e de prevenção da ocorrência de problemas relacionados ao trabalho.

Agrega comportamentos de não reclamação em caso de interrupção, de manutenção da atitude positiva até mesmo quando as coisas não acontecem da maneira esperada, de não ofender-se quando suas sugestões não são seguidas, de sacrifício de seu interesse pessoal pelo bem do grupo de trabalho e de não consideração da rejeição de suas ideias como ofensa pessoal.

Inclui comportamentos de promoção da organização externamente, protegendo-a e defendendo-a, e mantendo-se comprometido até em situações adversas.

Considera comportamentos de aderência a regras, regulamentações e procedimentos organizacionais até mesmo quando ninguém está observando ou monitorando seu cumprimento.

Inclui o engajamento em comportamentos relacionados à tarefa em nível tão extremo que os comportamentos chegam a ser reconhecidos como comportamentos voluntários.

Representa comportamentos de interesse ou comprometimento com a organização como um todo, incluindo a atuação em níveis de trabalho diferentes do seu.

Agrega comportamentos voluntários de busca por desenvolvimento de conhecimentos e habilidades.

\section{Moon et al. (2004)}

\section{Dimensões}

\section{Descrição}

Ajuda

Inclui comportamentos interpessoais voluntários de doação de tempo e/ou energia para apoiar colegas de trabalho.

Complacência

Congrega comportamentos de apoio e respeito a regras e regulamentações estabelecidas pela organização.

Agrega comportamentos interpessoais de redução ou prevenção de ocorrências afetivamente negativas no lugar de trabalho.

Considera comportamentos de promoção de mudança e melhora de produtos, processos, serviços, ideais e relacionamentos focados na organização.

Inovação

Por exemplo, a dimensão de Moon et al. (2004), que resulta da combinação entre promoção e interpessoal, e a de Podsakoff et al. (2000), denominada de comportamento de ajuda, podem ser consideradas justapostas à dimensão de cooperação com os colegas utilizada na literatura nacional. Isso também pode ser observado entre 
as dimensões lealdade e aquela resultante da combinação entre proteção e organizacional e a dimensão nacional de criação de clima favorável à organização no ambiente externo. Com relação à dimensão de autodesenvolvimento (Podsakoff et al., 2000), a sobreposição ocorre com a dimensão nacional de autotreinamento. Assim, é possível afirmar que há diferenças entre as dimensões encontradas na literatura nacional e as encontradas na estrangeira, mas parece haver mais semelhanças, o que é compreensível já que os modelos partem da mesma base teórica.

O periódico que mais agregou artigos foi a Revista Administração Contemporânea, com três dos artigos publicados (Tabela 5).

TABELA 5. Número e percentual de artigos publicados entre 1996 e 2013 com ao menos uma variável critério categorizada como comportamento de cidadania organizacional por periódico avaliado

\begin{tabular}{lc}
\hline Revista & $\mathbf{N}$ \\
\hline Revista Administração Contemporânea - RAC (ANPAD) & 3 \\
Psicologia: Organizações e Trabalho - rPOT (SBPOT) & 2 \\
Psicologia, Ciência e Profissão (CFP) & 1 \\
Estudos de Psicologia (UFRN) & 1 \\
Psicologia: Teoria e Pesquisa (UnB) & 1 \\
Total & $\mathbf{8}$
\end{tabular}

Considerando o ano de publicação, aquele com maior número de publicações foi o de 2003, em que dois artigos foram publicados. O ano faz parte do terceiro triênio de anos considerados na análise (2002-2004) que deteve o maior número de publicações (Figura 1). Em 11 dos anos considerados não houve publicação de artigos com uma ou mais dimensões de cidadania organizacional nas revistas avaliadas. O dado não agregado sugere que essa produção ainda é inconsistente e não está aumentando, ao contrário da produção internacional (Moon et al., 2004; Podsakoff et al., 2000). Além disso, no último triênio só foram produzidos artigos que consideravam subdimensões de cidadania organizacional, tal quais como concebidas por Porto e Tamayo (2003) e Siqueira (1995).

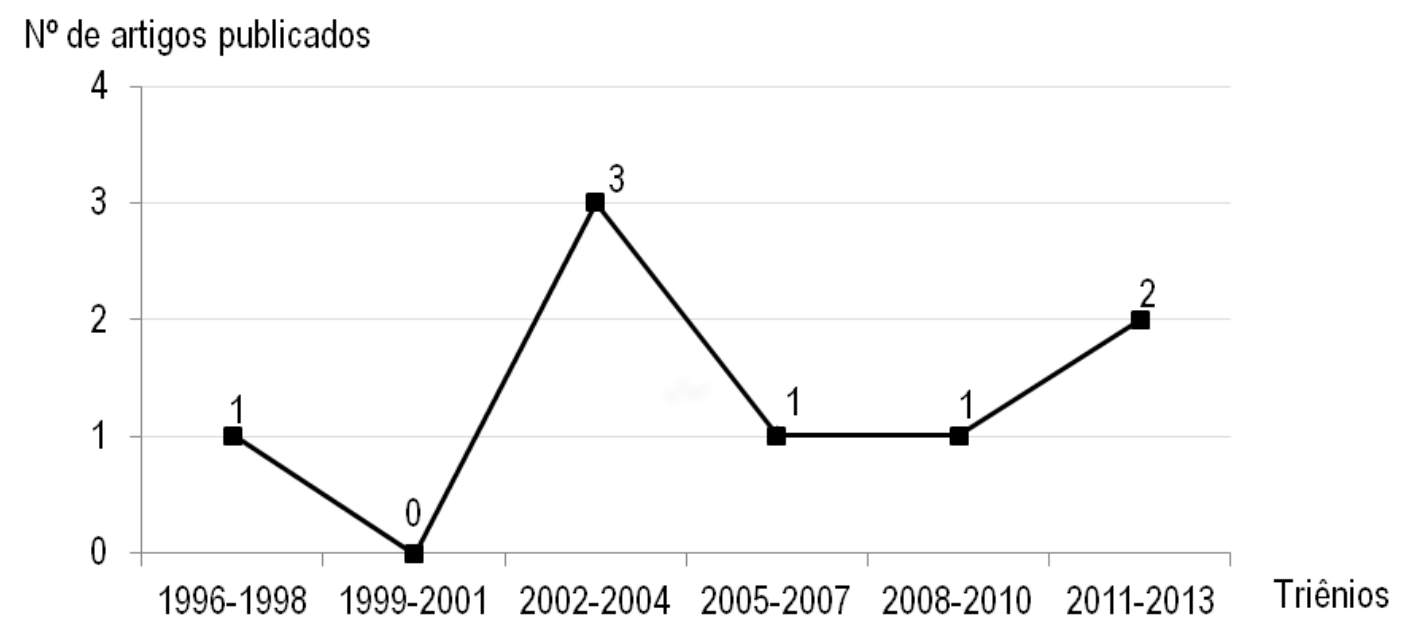

FIGURA 1. Número de artigos publicados entre 1996 e 2013 com ao menos uma variável critério categorizada como comportamento de cidadania organizacional por quadriênio.

Entre as 14 filiações institucionais de autores visíveis nos artigos, sete eram da Universidade de Brasília e dois da Pontifícia Universidade Católica do Paraná, dado que sugere a existência de um núcleo voltado para o estudo de cidadania organizacional nessas universidades. Apenas um vínculo com a pós-graduação foi 
visualizado nos artigos avaliados, e talvez isso se deva a não revelação dessa informação por parte dos autores ao elaborarem suas apresentações para os periódicos.

Seis dos artigos analisados também avaliaram outras variáveis de comportamento organizacional que não cidadania organizacional, sendo que alguns deles apresentaram mais de duas variáveis diferentes em seu escopo. As variáveis também estudadas foram: valores (cultura organizacional); satisfação (afeto no trabalho); comprometimento ou envolvimento ou lealdade e reciprocidade organizacional (contratos psicológicos); crenças sobre mudanças, percepção de mudanças e resistência (atitudes frente a mudanças organizacionais); e percepção de suporte (cognição no trabalho). Todas as variáveis assinaladas foram consideradas antecedentes de comportamentos de cidadania organizacional nos estudos.

Coincidentemente ou não, dois dos estudos nacionais incluíram como antecedentes comprometimento, envolvimento ou lealdade e/ou satisfação, que são variáveis categorizadas por Podsakoff et al. (2000) como características individuais do empregado, as mais estudadas como antecedentes de cidadania organizacional na literatura internacional. A única exceção foi o estudo de valores como antecedente em três artigos. Variáveis consequentes não foram visualizadas nos estudos avaliados, o que impossibilitou qualquer tipo de comparação com pesquisas estrangeiras.

A maior parte das pesquisas (seis dos oito artigos) teve a finalidade de gerar conhecimento, enquanto o restante, além de gerá-lo, também buscou gerar instrumento ou apenas gerar instrumento. O dado assemelha-se àquele obtido por Borges-Andrade e Pagotto (2010) para a literatura geral em comportamento organizacional, uma vez que a maioria dos artigos objetivou gerar conhecimento. Todos apresentaram desenho de pesquisa por survey. Ademais, ao contrário da literatura nacional em comportamento organizacional, em que o percentual de artigos utilizando a técnica quantitativa era semelhante ao de artigos que utilizavam a qualitativa, a totalidade dos artigos sobre cidadania organizacional descreveu técnicas quantitativas.

Quanto às amostras, seis artigos utilizaram de várias organizações, e o restante, de uma organização. Os dados sugerem que a maioria das pesquisas na área são generalizáveis para diferentes organizações, uma vez que basearam suas análises em organizações distintas. A maioria, sete de oito artigos, apropriou-se de dados de origem primária. De certa forma, os dados sugerem que as organizações ainda não possuem bancos rotineiramente atualizados com dados de cidadania organizacional. Uma atualização contínua de dados serviria à própria organização para monitoramento dos comportamentos e aos pesquisadores externos que desejassem fomentar a área.

Quanto ao procedimento de coleta de dados, todas as pesquisas utilizaram escalas e questionários. Parece haver menor variabilidade das técnicas utilizadas que a da produção nacional em comportamento organizacional (Borges-Andrade \& Pagotto, 2010), com grande peso de escalas e questionários respondidos pelo próprio funcionário que avaliam de forma indireta o construto cidadania organizacional. Esse procedimento difere do que vem sendo adotado na literatura estrangeira, que privilegia medidas de múltiplas fontes (Dalal, 2005; Podsakoff et al., 2009).

Entre as técnicas de análise de dados, oito em 10 técnicas relatadas foram inferenciais. Considerando as pesquisas com análises inferenciais, as técnicas de análise fatorial exploratória, correlação e regressão múltipla foram as mais utilizadas, cada uma em cinco estudos diferentes (Tabela 6). Além disso, um dos artigos utilizou a técnica de modelagem por equação estrutural. Os dados sugerem que os estudos nacionais estão buscando a predição do fenômeno, como já observado na literatura internacional (Podsakoff et al., 2000). Chama atenção o maior número de pesquisas utilizando técnicas inferenciais no campo de cidadania organizacional em comparação ao campo de microcomportamento organizacional no Brasil (Borges-Andrade \& Pagotto, 2010).

TABELA 6. Procedimentos de análise de dados inferenciais utilizados nas pesquisas sobre comportamentos de cidadania organizacional relatadas em seis periódicos nacionais de administração e psicologia

\begin{tabular}{lcc}
\hline Técnica inferencial utilizada & N & \% \\
Análise fatorial exploratória & 5 & 25.0 \\
Correlação & 5 & 25.0 \\
Regressão múltipla & 5 & 25.0
\end{tabular}


Os setores públicos e privados da economia foram considerados nas pesquisas, 50\% e 33\%, respectivamente. Por fim, 75\% dos artigos das pesquisas ocuparam-se de dados provenientes do segmento terciário, sendo, portanto, o contexto do setor de serviços em que a maior parte das pesquisas sobre cidadania organizacional vem sendo realizada. Uma pesquisa também reportou coleta de dados junto ao setor secundário. As tendências quanto aos setores e aos segmentos da economia foram semelhantes àquelas apresentadas por Borges-Andrade e Pagotto (2010), com o setor público seguido pelo privado.

\section{DISCUSSÃO}

Entre os problemas apresentados pela literatura internacional em cidadania organizacional, destaca-se a não delimitação do construto e, como consequência, o estudo de uma gama de elementos mal definidos. Além disso, alguns elementos são mais estudados que outros, o que gera falta de conhecimento acerca de alguns componentes da cidadania organizacional, como inovação (Moon et al., 2004), e dificulta a compreensão global sobre o fenômeno.

Somados aos problemas enfrentados internacionalmente pela área, estão alguns enfrentados nacionalmente. A produção brasileira na área é muito pequena, chegando, inclusive, à ausência de publicação de artigos em 11 dos anos de revisão avaliados. Adicionalmente, nenhum dos estudos avaliados ocupa-se da análise de consequentes da cidadania organizacional, o que se contrapõe a estudos da literatura estrangeira, como pode ser visualizado em metanálises (Dalal, 2005; Nielsen et al., 2009; Podsakoff et al., 2009). A falta desses estudos pode impactar na defesa da relevância desse construto e, por conseguinte, influenciar na falta de interesse pelo tema entre pesquisadores nacionais.

Quanto à localização da produção nacional, apenas cinco entre os 14 periódicos avaliados apresentaram artigos tratando de comportamentos de cidadania organizacional, o que sugere a pequena dispersão da subárea. Do número de instituições às quais os autores eram afiliados, apenas sete, de 1996 a 2013, corrobora o observado. Os dados parecem preocupantes, uma vez que mostram que o estudo de cidadania organizacional no Brasil parece estagnado e limitado, não seguindo a tendência internacional na área. Uma vez que a variável vem sendo ligada a diversas outras do contexto organizacional, como desempenho individual e organizacional, seu estudo é de suma importância para a compreensão da dinâmica organizacional (Dalal, 2005; Nielsen et al., 2009; Podsakoff et al., 2009).

Assim, fazem parte da agenda de pesquisa sobre comportamentos de cidadania organizacional a realização de estudos que considerem a natureza multidimensional do construto e que avaliem seu impacto em resultados organizacionais e a publicação de um maior volume de pesquisas, produzidas por diferentes instituições e publicadas por diferentes periódicos. Para tanto, é importante e imprescindível a associação de grupos de pesquisa, com ramificações nas áreas de administração e psicologia.

Finalmente, sugere-se a realização de estudos com técnicas observacionais que abarquem os comportamentos de cidadania per se e que utilizem diferentes fontes de informação e não somente autorrelatos, além de estudos experimentais e quasi-experimentais que possibilitem o estabelecimento de cadeias de causalidade entre cidadania organizacional e seus antecedentes e consequentes. Adicionalmente, sugere-se a realização de pesquisas longitudinais sobre o processo de desenvolvimento de comportamentos de cidadania organizacional do funcionário ao longo de sua carreira na organização. 
Este estudo refletiu a revisão da literatura em um período definido que, embora abarque mais de 15 anos, não compreende o período total de existência do construto. Além disso, o fato de a cidadania organizacional possuir uma série de dimensões ainda não tão bem definidas pode ter dificultado a detecção de artigos sob sua alçada. Por fim, uma metanálise de artigos com microvariáveis de cidadania organizacional publicada na literatura nacional seria desejável. Esse intento é, no entanto, impedido pela ausência de um número maior de artigos. Entre os oito artigos revisados aqui, dois não abarcavam ou mencionavam cidadania organizacional como um todo. Isso remete à necessidade de mais pesquisas que considerem cidadania organizacional de forma geral e sob o nível de análise micro.

Antes que seu estudo cresça de maneira sistemática no contexto nacional, será temerário prescrever a profissionais e gerentes o que fazer para alterar indicadores de cidadania organizacional. Essa prescrição, com base na suposição de que os achados internacionais se aplicam ao Brasil, não se sustenta com argumentos ou de forma empírica, especialmente se são levadas em conta as evidências sobre a investigação transcultural de comportamento organizacional (Gelfand et al., 2007).

\section{REFERÊNCIAS}

Borges-Andrade, J. E., \& Pagotto, C. P. (2010). O estado da arte da pesquisa brasileira em Psicologia do Trabalho e Organizacional. Psicologia: Teoria e Pesquisa, 26(Número Especial), 37-50.

Borman, W. C., Penner, L. A., Allen, T. D., \& Motowidlo, S. J. (2001). Personality predictors of citizenship performance. International Journal of Selection and Assessment, 9(1/2), 52-69. doi: 10.1111/1468-2389.00163

Chang, C. H., Johnson, R. E., \& Yang, L. Q. (2007). Emotional strain and organizational citizenship behaviours: A metaanalysis and review. Work \& Stress: An International Journal of Work, Health \& Organisations, 21(4), 312-332. doi: $10.1080 / 02678370701758124$

Chiaburu, D. S., Oh, I. S., Berry, C. M., Li, N., \& Gardner, R. G. (2011). The five-factor model of personality traits and organizational citizenship behaviors: A meta-analysis. Journal of Applied Psychology, 96(6), 1140-1166. doi: 10.1037/ a0024004

Dalal, R. S. (2005). A meta-analysis of the relationship between organizational citizenship behavior and counterproductive work behavior. Journal of Applied Psychology, 90(6), 1241-1241. doi: 10.1037/0021-9010.90.6.1241

Eatough, E. M., Chang, C. H., Miloslavic, S. A., \& Johnson, R. E. (2011). Relationships of role stressors with organizational citizenship behavior: A meta-analysis. Journal of Applied Psychology, 96(3), 619-632. doi: 10.1037/a0021887

Gelfand, M. J., Erez, M., \& Aycan, Z. (2007). Cross-cultural organizational behavior. Annual Review of Psychology, 58, 479-514. doi: 10.1146/annurev.psych.58.110405.085559

Hopkins, M. (2003). The planetary bargain - Corporate social responsibility matters. Londres: Earthscan.

Hopkins, M. (2005). Measurement of corporate social responsibility. International Journal of Management \& Decision Making, 6(3/4), 213-231. doi: 10.1504/IJMDM.2005.006549

Ilies, R., Nahrgang, J. D., \& Morgeson, F. P. (2007). Leader-member exchange and citizenship behaviors: A meta-analysis. Journal of Applied Psychology, 92(1), 269-277. doi: 10.1037/0021-9010.92.1.269

Katz, D., \& Kahn, R. L. (1966). The social psychology of organizations. Hoboken, NJ: John Wiley \& Sons.

LePine, J. A., Erez, A., \& Johnson, D. E. (2002). The nature of dimensionality of organizational citizenship behavior: A critical review and meta-analysis. Journal of Applied Psychology, 87(1), 52-65. doi: 10.1037//0021-9010.87.1.52

Moon, H., Van Dyne, L., \& Wrobel, K. (2004). The circumplex model and the future of organizational citizenship behavior research. In D. L. Turnipseed (Ed.), Handbook of organizational citizenship behavior (pp. 3-23). Nova York: Nova Science Publishers.

Nielsen, T. M., Hrivnak, G. A., \& Shaw, M. (2009). Utility of OCB: Organizational citizenship behavior and group performance in a resource allocation framework. Small Group Research, 40(5), 555-577. doi: 10.1177/1046496409339630

Podsakoff, P. M., MacKenzie, S. B., Paine, J. B., \& Bachrach, D. G. (2000). Organizational citizenship behaviors: A critical review of the theoretical and empirical literature and suggestions for future research. Journal of Management, 26(3), 513-563. doi: $10.1177 / 014920630002600307$

Podsakoff, N. P., Witing, S. W., Podsakoff, P. M., \& Blume, B. D. (2009). Individual-and organizational-level consequences of organizational citizenship behaviors: A meta-analysis. Journal of Applied Psychology, 94(1), 122-141. doi: 10.1037/ a0013079 
Pond, S. B., Nacoste, R. W., Mohr, M. F., \& Rodriguez, C. M. (1997). The measurement of organizational citizenship behavior: Are we assuming too much? Journal of Applied Social Psychology, 27(17), 1527-1544. doi: 10.1111/j.1559-1816.1997.tb01611.x

Porto, J. B., \& Tamayo, A. (2003). Desenvolvimento e validação da escala de civismo nas organizações. Estudos de Psicologia (Natal), 8(3), 393-402.

Rego, A. (2002). Do que falamos quando falamos de comportamentos de cidadania organizacional? In A. Rego (Org.), Comportamentos de cidadania nas organizações (pp. 1-47). Alfragide: McGraw-Hill.

Siqueira, M. M. M. (1995). Antecedentes de comportamentos de cidadania organizacional: A análise de um modelo pós-cognitivo (Tese de doutorado não publicada). Universidade de Brasília, Brasília.

Siqueira, M. M. M. (2002). Medidas do comportamento organizacional. Estudos de Psicologia, 7(Número Especial), 11-18.

Staw, B. M. (1984). Organizational behavior: A review and reformulation of the field's outcome variables. Annual Review of Psychology, 35, 627-666.

Van Dyne, L., Vandewalle, D., Kostova, T., Latham, M. E., \& Cummings, L. L. (2000). Collectivism, propensity to trust and selfesteem as predictors of organizational citizenship in a non-work setting. Journal of Organizational Behavior, 21(1), 3-23. doi: 10.1002/(SICl)1099-1379(200002)

Zellars, K. L., Tepper, B. J., \& Duffy M. K. (2002). Abusive supervision and subordinates' organizational citizenship behavior. Journal of Applied Psychology, 87(6), 1068-1076. doi: 10.1037//0021-9010.87.6.1068 